\title{
Cognitive-behavioral family therapy as psychoeducation for adolescents with high- functioning autism spectrum disorders: Aware and Care for my Autistic Traits (ACAT) program study protocol for a pragmatic multisite randomized controlled trial
}

Fumiyo Oshima ${ }^{1 *}$, Mandy William², Noriko Takahashi ${ }^{3}$, Aki Tsuchiyagaito ${ }^{1,4}$, Hitoshi Kuwabara ${ }^{5}$, Akihiro Shiina ${ }^{6}$, Mikuko Seto ${ }^{1}$, Minako Hongo ${ }^{1}$, Yui Iwama ${ }^{1}$, Yoshiyuki Hirano ${ }^{1}$, Chihiro Sutoh ${ }^{1}$, Kayoko Taguchi ${ }^{1}$, Tokiko Yoshida ${ }^{1}$, Yohei Kawasaki, Yoshihito Ozawa”7, Jiro Masuya ${ }^{3,8}$, Noriyuki Sato $^{3}$, Shizuka Nakamura ${ }^{3}$, Masaru Kuno ${ }^{1}$, Jumpei Takahashi ${ }^{1}$, Toshiyuki Ohtani ${ }^{1}$, Daisuke Matsuzawa ${ }^{1}$, Naoko Inada ${ }^{9}$, Miho Kuroda ${ }^{10}$, Mika Ando ${ }^{11}$, Arinobu Hori ${ }^{12}$, Akiko Nakagawa ${ }^{1}$ and Eiji Shimizu

\footnotetext{
Abstract

Background: One aim of an autism spectrum disorder (ASD) diagnosis is to obtain special support for the disorder, though this does not guarantee practical support. We developed a psychoeducational program using cognitivebehavioral therapy (CBT) and Aware and Care for my Autistic Traits (ACAT) for Japanese adolescents with highfunctioning ASD and their parents.

(Continued on next page)
}

\footnotetext{
* Correspondence: fumiyooshima@gmail.com

${ }^{1}$ Research Center for Child Mental Development, Chiba University, 1-8-1 Inohana Chuouku, Chiba 260-8670, Japan

Full list of author information is available at the end of the article
}

(c) The Author(s). 2020 Open Access This article is licensed under a Creative Commons Attribution 4.0 International License, which permits use, sharing, adaptation, distribution and reproduction in any medium or format, as long as you give appropriate credit to the original author(s) and the source, provide a link to the Creative Commons licence, and indicate if changes were made. The images or other third party material in this article are included in the article's Creative Commons licence, unless indicated otherwise in a credit line to the material. If material is not included in the article's Creative Commons licence and your intended use is not permitted by statutory regulation or exceeds the permitted use, you will need to obtain permission directly from the copyright holder. To view a copy of this licence, visit http:/creativecommons.org/licenses/by/4.0/. The Creative Commons Public Domain Dedication waiver (http://creativecommons.org/publicdomain/zero/1.0/) applies to the data made available in this article, unless otherwise stated in a credit line to the data. 
(Continued from previous page)

Methods: This multisite study is a randomized controlled trial. In total, 24 participants will be assigned to the ACAT group and 24 to the treatment-as-usual (TAU) group. The ACAT group will receive a weekly 100-min session for 6 weeks, regular medical care, and one follow-up session. In this ongoing clinical trial, we will compare the scores of the measures recorded in the pre- and post-intervention stages between the ACAT and TAU groups. A total of 41 patients out of a target of 48 have participated in the trial to date. The primary outcome measure is the Autism Knowledge Questionnaire. Secondary outcome measures include Barriers to Access to Care Evaluation 3rd Edition, the Strengths and Difficulties Questionnaire, the Vineland Adaptive Behavior Scales second edition, the Parenting Resilience Elements Questionnaire, the General Health Questionnaire 12, and the Depression Self-Rating Scale for Children assessments, as well as an electroencephalographic recording.

Discussion: It is expected that participants in the ACAT group will significantly increase their self-understanding and awareness of ASD symptoms compared to those in the TAU group. Additionally, the ACAT group is expected to exhibit improved social adaptation and mental health if children and parents are able to better understand the ASD characteristics through sessions. This intervention will contribute to the establishment of an effective evidencebased treatment strategy for adolescents with ASD.

Trial registration: UMIN Register 000029851. Registered on January 06, 2018

Keywords: High-functioning autism spectrum disorder, Randomized controlled trial, Cognitive-behavioral family therapy, Psychoeducation

\section{Background}

Autism spectrum disorder (ASD) is a pervasive neurodevelopmental disorder characterized by impairments in social communication and restricted, repetitive patterns of behavior, interests, or activities [1]. ASD is a relatively common neurodevelopmental condition that affects $1-1.5 \%$ of the population [2, 3]. Individuals with high-functioning ASD (HF-ASD) have normal-range IQs and extensive verbal abilities and usually attend regular schools. Nevertheless, their difficulties are potentially serious and can include impaired social relations, obsessions, and uneven levels of intellectual and cognitive functioning [4]. When patients with HF-ASD become independent and increase social interactions as they enter adolescence, they become aware of their differences to their surroundings, which tends to lower their self-efficacy and self-esteem and increase feelings of depression and anxiety [5-7].

An extensive treatment has recently been made available to support the central characteristics of ASD early in life, as evidenced by the Early Start Denver Model [8] and parent-mediated communication-focused treatment in children with autism (PACT) [9]. These treatment programs are not available for people with autism post-early childhood. However, individuals with autism who are diagnosed later may experience benefits in social functioning by gaining support, such as social skills training [10, 11] and/or reasonable accommodation [12].

In childhood and adolescence, individuals without knowledge of focal features and those with HF-ASD can easily experience self-stigma such as feeling "abnormal" or "inferior" [13]. As stigma acts as a barrier to help-seeking behavior [14], patients may experience reduced opportunities to obtain support alongside increased feelings of experiencing difficulties [15].

Conversely, the provision of psychoeducation on ASD has been reported to decrease adverse feelings toward children with the disorder. Gaining knowledge on ASD is also extremely important to the parents. Farrugia reported that parents who gained medical knowledge about ASD experienced decreased stigma toward children with ASD [16]. In addition, Gordon et al. [17] demonstrated that participation in a brief parent-child program of psychoeducation, the PsychoEducational Groups for Autism Spectrum Understanding and Support (PEGASUS), which sought to promote selfunderstanding and acceptance of HF-ASD, increased self-awareness in both parents and children. The PEGA SUS is a psychoeducation group for young individuals with HF-ASD and their parents that promotes awareness of autistic traits. However, it cannot help them cope with behavioral problems in multiple domains. Therefore, we conjectured that cognitive-behavioral family therapy (family CBT) may help HF-ASD children/adolescents and their families to understand the central characteristics of ASD and cope with the cognitive and behavioral problems caused by autistic traits. The CBT treatment seeks to produce cognitive alterations in various ways to endure emotional and behavioral changes [18]. Reports have suggested that the application of CBT for ASD represents a major advance in the treatment of cooccurring anxiety and depression in HF-ASD patients [6, $19,20]$. There is a small body of literature indicating that CBT is effective for managing problems faced by young individuals with ASD, such as anger, anxiety, social interaction, and emotional understanding [21]. For 
children with ASD, modifying the standard CBT protocol is necessary to induce general and permanent changes [22]. In this regard, we have included family participation because the parents need to understand the distinct features of their children's AD to understand the considerations required when caring for them.

For this study, the authors developed a new intervention for HF-ASD children/adolescents termed the Aware and Care for my Autistic Traits (ACAT) program. This program includes psychoeducation and family CBT for HF-ASD children/adolescents and their parents/guardians. The details of this program are explained in the "Methods/design" section.

Since the ACAT program is based on psychoeducation that augments family CBT, the primary outcome is the increased awareness of ASD measured by the child/ youth version of the Autism Knowledge Quiz (AKQ) [17]. In addition, a social adaptation scale and a stigma scale are collected as secondary outcomes to monitor the relative improvement of these two factors. Moreover, investigating the relationship between behavioral and neural changes after the intervention can provide strong evidence that the intervention has an impact on behavioral and neural substrates. Therefore, we will record resting-state electroencephalograms (EEG) as a neural outcome in this study for exploratory purposes. Although the causes and biomarkers of ASD remain unclear, evidence suggests that ASD is a neurobiological disorder underscored by abnormal brain activity related to the observed social deficits [23]. EEG, which primarily measures neurophysiological changes related to postsynaptic activity over the cortex, can capture and characterize abnormal brain activity. For example, individuals with ASD showed increased short-range connections in the lateral-frontal electrodes and decreased long-range connections in the fronto-occipital electrodes compared with healthy individuals [24]. Moreover, other studies have shown that altered EEG patterns are normalized in response to improved social behaviors after social training programs, which indicates that psychosocial interventions have the potential to alter brain activity as measured by EEG.

\section{Objectives}

This study aims to examine the efficacy of the newly developed psychoeducation program for parents and individuals with HF-ASD, or ACAT, by comparing with the treatment-as-usual (TAU) group. Individuals and parents who are assigned to the ACAT group will keep their current treatments, which the ACAT program will augment. We hypothesize that the ACAT group will show greater children and parents'/guardians' understanding and awareness of their autistic attributes than the TAU group. Randomized controlled trials will be conducted at multiple sites to investigate the relative efficacy of ACAT compared to TAU.

Our goal is to examine the relative efficacy of the combination of psychoeducation and family CBT for both child/adolescent patients with HF-ASD and their parents/ guardians after diagnosis. This study will help to establish a new evidence-based psychoeducational treatment strategy for child/adolescent patients with HF-ASD and their parents/guardians. It will enable more treatment options as an after-diagnosis service and improve the dissemination of psychoeducation and CBT for HF-ASD.

\section{Methods/design \\ Study design}

This study is designed as a prospective randomized open-label endpoint trial with two parallel intervention groups consisting of a 6-week treatment of treatmentas-usual (TAU group) alone and an ACAT combined with TAU (ACAT group). It is randomized, multisite, stratified, single-blinded, and is conducted through a parallel between-group design.

Qualified individuals who consent to this trial are randomly assigned to a TAU sustained group or a COMB group that combines psychoeducation and family CBT. The study is planned in accordance with the Standard and Protocol Items: Recommendations for Interventional Trials [25] and will be analyzed and reported in line with the Consolidated Standards of Reporting Trials Recommendations [26].

\section{Participants}

The eligible participants in the current study are individuals diagnosed with ASD and their parents or guardians. Before the final inclusion, both written and verbal informed consent is obtained from all participants in the study.

\section{Inclusion criteria}

The participants in this study comprise patients and their parents/guardians who meet the following criteria during the screening process:

\section{Individual child/adolescent with ASD:}

1. Meet the cutoff value for ASD measured using the Autism Diagnostic Observation Schedule [27] or Autism Diagnostic Interview-Revised [28]

2. Have a verbal IQ of 90 or above as measured by the Wechsler Intelligence Scale for Children [29]

3. Have a social difficulty assessment of "some need" or higher measured by the Strengths and Difficulties Questionnaires [30]

4. Aged between 10 and 17 years at the time of consent, with one of the parents being eligible to participate in psychoeducation and CBT (if assigned to $\mathrm{COMB}$ ) 
5. Diagnosed with ASD by a primary doctor or other doctors or rehabilitation center for children

6. Received outpatient psychiatric care

7. Able to understand CBT and is mentally and physically able to sustain therapy for at least 2 months

\section{Parents/guardians of ASD individuals:}

1. Living with the ASD individual as a parent or a grandparent and acts as a guardian

2. Able to participate in CBT once a week with the ASD individual

\section{Exclusion criteria}

Individual child/adolescent with ASD:

1. Current active suicidal intent

2. Active repetitive antisocial behavior

3. Active severe degenerative physical disorder that may disrupt CBT

\section{Parents/guardians of ASD individuals:}

1) Must not meet the diagnostic criteria for psychiatric disorders measured by the MiniInternational Neuropsychiatric Interview [31] to be eligible for participation in the study

2) Unable to accompany the patient to the sessions

\section{Procedures}

Beginning in March 2018, recruitment, treatment, and data collection are being conducted in two cities in Japan (Chiba and Fukushima). The last follow-up treatment will be completed in August 2020. Children and adolescent outpatients (10-17 years of age) diagnosed with ASD and their parents and guardians are informed about the intervention in waiting rooms at Chiba University Hospital and a local psychiatry clinic. For those who show interest, more information is provided by the recruitment staff.

The flow chart of this clinical trial is shown in Fig. 1. The trial will be conducted over 6 weeks of interventions and 4 weeks of follow-up. To evaluate treatment efficacy and safety, outcome measure assessments will be administered before and after the interventions. The participants assigned to $\mathrm{COMB}$ will continue their usual treatment with their primary doctors and also participate in six sessions of the ACAT program (a 100-min psychoeducation and family CBT program developed by the authors) every week (the ACAT program is conducted for a duration of 6 weeks). Participants taking psychotropic drugs regularly as medical treatment will be requested to remain on stable dosage during the interventions; however, the dosage may change if their primary or prescribing physicians consider it medically necessary. Any change in the dosage will be recorded in the list of daily doses of psychoactive drugs. A follow-up interview will be conducted 4 weeks after the completion of the interventions (10th week). Interviews and

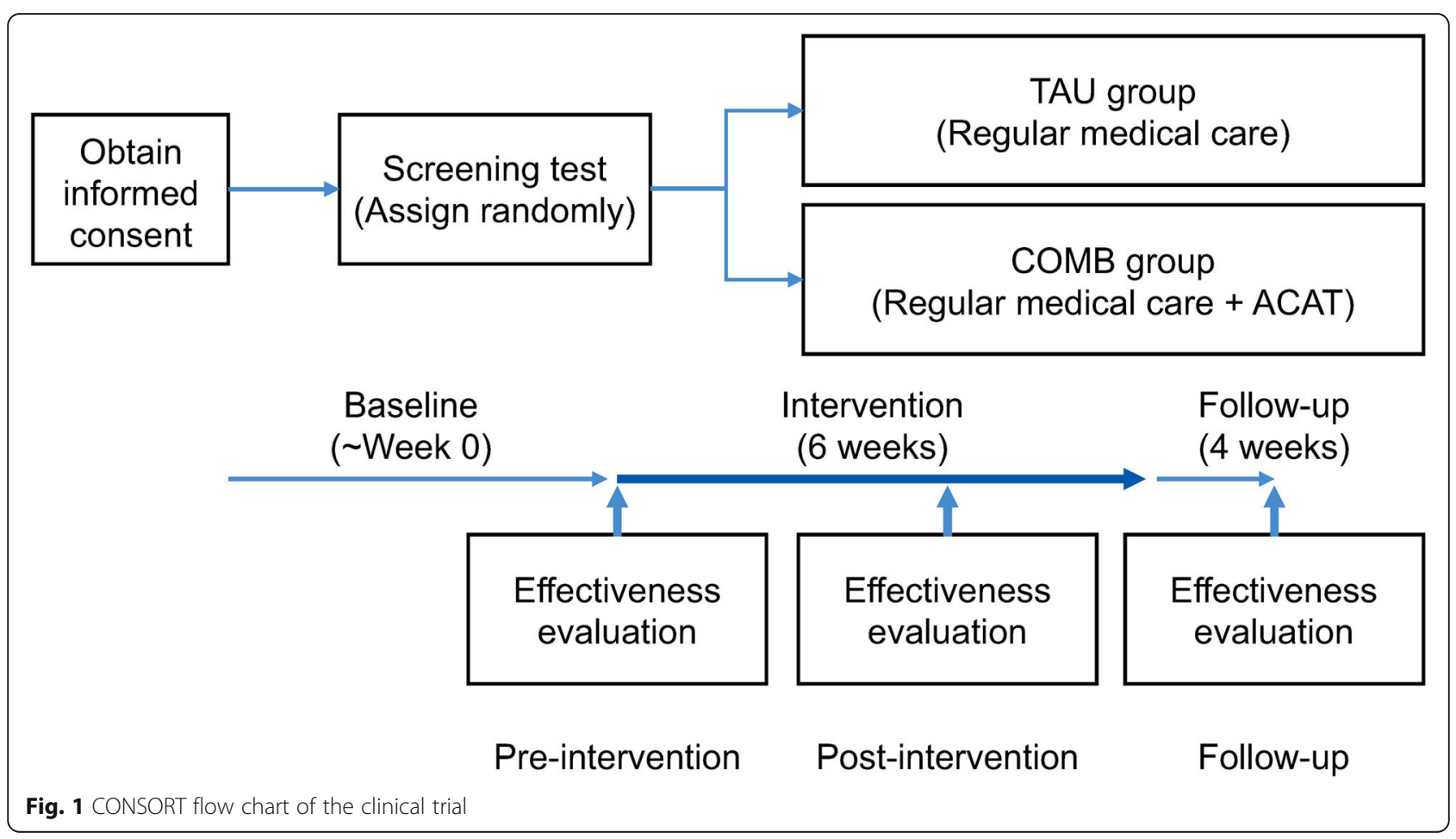


symptom assessments will be undertaken at each facility during week 0 (pre-intervention), week 6 (post-intervention), and week 10 (follow-up). The participants assigned to the TAU group will be required to continue their usual treatment with their primary doctors. Furthermore, a combination of the following treatments is prohibited with their usual treatment: psychotherapy that conforms to CBT or physiological therapies, including electroconvulsive therapy and magnetic stimulation therapy. The interviews and symptom assessments at preintervention, post-intervention, and follow-up will be conducted for TAU at each facility in the same manner as that for COMB. The restrictions for psychotropic drugs are the same as those for the COMB group. All sessions and psychological examinations are free for the participant. Both child/adolescent patients and their parents/guardians will receive 10,000 yen (about 100 USD) after completion of the last measurement. Any harm that occurs will be reported after the study period, whether or not it could have been related to the study treatment. Patients will be observed for up to 4 weeks (after discontinuation). To date, 41 patients in total have been randomized.

\section{Randomization and blinding}

Enrollment and randomization are based on the central registration system of the Clinical Research Data Center at Chiba University Hospital, Chiba, Japan. Two allocation factors are used for stratification: the degree of knowledge (high or low) regarding the characteristics of ASD according to the primary outcome measure, the Autism Knowledge Quiz-Child (AKQ-C), and gender (male or female). At the end of the baseline assessment, eligible participants will be randomly assigned to either the TAU arm or ACAT plus TAU arm at a ratio of 1:1, with assignments made using the minimization method, ensuring a balance across baseline AKQ scores and gender. Each participant will then be assigned to one of the two treatments. Participants will not be blinded to the group to which they are assigned before consenting to participate in the study. However, trained testers, who will assess all outcome measures except the selfassessment scale, will be blinded to the group.

\section{Data monitoring}

An Independent Data Monitoring Committee has been established for this study. It will meet every 3 months to review a report created by the researchers to monitor the progress of recruitment and data collection (e.g., completion rates for each follow-up).

\section{Ensuring the quality of therapists' skills and sessions}

Psychiatrists and clinical psychologists, licensed for at least 3 years and involved in clinical work with ASD individuals, have been engaged as cognitive-behavioral therapists for this study. The therapists for ACAT received at least $6 \mathrm{~h}$ of initial training on the intervention, read the treatment manual, listened to the audiotape set of the model therapist performing the treatment, and are supervised weekly by the clinical supervisor (the first author of this thesis who developed the ACAT protocol). All sessions will be recorded and evaluated by researchers unrelated to the development or operation of the ACAT program using the Cognitive Therapy Scale [32].

\section{ACAT program details}

This study follows the ACAT protocol shown in Table 1. We started to develop a psychological education program for ASD, referring to programs for ASD outside of Japan that can be adopted as the standard in Japan.

The concept of the program is to help adolescents with ASD and their caregivers learn about ASD and improve their skills in coping with "ASD traits" using CBT. The mechanism of the expected effect is that it will increase and consequently eliminate social and psychological difficulties. The ACAT protocol was preliminarily utilized in two patients with ASD and their parents to test it in clinical situations and collect feedback, after which it was revised. The ACAT program is conducted over a 100-min session per week with a 10-min break, with six sessions in total. An individual with ASD and a parent/guardian attends the session together. An agenda is prepared for each session (Table 2). The workflow is described in Table 1. The first three sessions focus on participants learning about their own ASD characteristics and their ability to externalize these characteristics by creating funny nicknames, which facilitates selfmonitoring skills and development of metacognition of their characteristics. From the fourth session onward, featured difficulties (symptoms) arising from their autistic traits are discussed. These procedures reasonably accommodate the individual abilities of each participant. Each session has a homework assignment that focuses on reviewing the discussed material. If a participant forgets to do a homework assignment or is absent or late to a session for any reason, a make-up session will be provided to fill in the gaps.

\section{Schedule for screening tests}

Table 3 describes the operational schedule for the screening test and outcome measures.

Screening tests will be conducted for participants and their parents after obtaining their informed consent. A physician or therapist will perform the screening tests as shown in Table 3. Patients who qualify according to the selection criteria and do not meet the exclusion criteria will be selected. As inspection items, we will ascertain the backgrounds and names of participants and dosage 
Table 1 Agenda and purpose of each ACAT session

\begin{tabular}{|c|c|c|}
\hline $\begin{array}{l}\text { CBT } \\
\text { sessions }\end{array}$ & Session content & Session goals \\
\hline $\begin{array}{l}\text { Pre- } \\
\text { session }\end{array}$ & Learn about your psychological assessment for ASD & $\begin{array}{l}\text { The participants will learn about their personal characteristics and } \\
\text { autistic traits through the results of assessment tests. }\end{array}$ \\
\hline 1st CBT & Learn about the characteristics of your autistic traits & $\begin{array}{l}\text { The participants will understand the differences between autistic } \\
\text { attributes and typical developmental features. }\end{array}$ \\
\hline 2nd CBT & $\begin{array}{l}\text { Cognitive changes: learn not only about the "weakness" but also } \\
\text { "strengths" of your autistic traits }\end{array}$ & $\begin{array}{l}\text { The participants will discover the "strengths" of ASD, setting aside the } \\
\text { "weaknesses" and turning negative perspectives into rational neutral } \\
\text { understanding about themselves. }\end{array}$ \\
\hline 3rd CBT & $\begin{array}{l}\text { Cognitive changes: create "funny nicknames" for your autistic } \\
\text { traits }\end{array}$ & $\begin{array}{l}\text { The participants will learn to externalize their autistic characteristics, } \\
\text { facilitate self-monitoring skills, and develop metacognition. }\end{array}$ \\
\hline 4th CBT & $\begin{array}{l}\text { Behavioral changes: create a problem-solving plan for your autis- } \\
\text { tic traits }\end{array}$ & $\begin{array}{l}\text { The participants will learn how they can change and adjust their } \\
\text { problematic behaviors to cope with their autistic traits. }\end{array}$ \\
\hline 5th CBT & $\begin{array}{l}\text { Behavioral changes: plan a functional way to solve easy } \\
\text { problems that are caused by your autistic traits with your parents }\end{array}$ & $\begin{array}{l}\text { The participants will make problem-solving plans for their own autistic } \\
\text { traits and will also consider how to cope with problems when they } \\
\text { execute their plans. They will start with easier problems related to their } \\
\text { ASD traits. They will conduct these plans as a homework assignment. }\end{array}$ \\
\hline 6th CBT & $\begin{array}{l}\text { Behavioral changes: plan a functional way to solve more } \\
\text { complicated problems that are caused by your autistic traits with } \\
\text { your parents }\end{array}$ & $\begin{array}{l}\text { The participants will make problem-solving plans for their own autistic } \\
\text { traits and will also consider how to manage more complicated prob- } \\
\text { lems when they execute their plans. They will conduct these plans as a } \\
\text { homework assignment (this homework assignment will be checked at } \\
\text { a follow-up session). }\end{array}$ \\
\hline $\begin{array}{l}\text { CBT } \\
\text { follow- } \\
\text { up }\end{array}$ & Check how well you are living with your autistic traits & $\begin{array}{l}\text { The participants will review all skills and techniques learned to date. } \\
\text { They will check their understanding of their own autistic traits and } \\
\text { discuss the progress made in therapy, areas of continued effort, and } \\
\text { ongoing challenges. }\end{array}$ \\
\hline
\end{tabular}

of psychotropic drugs administered to them along with their usage period. We will conduct the Autism Diagnostic Observation Schedule Second Edition (ADOS-2) [27] and Autism Diagnostic Interview-Revised (ADI-R) [28] to assess ASD and the ADHD Rating Scale-IV (ADHD-RS) [33] to assess hyperactivity and impulsivity accompanying their ASD. We will conduct the Japanese version of the Sensory Profile [34] to assess dysesthesia and administer the Strengths and Difficulties Questionnaire (SDQ) [30] to assess behavioral problems. We will also conduct the Wechsler Adult Intelligence Scale-III (WAIS-III) or Wechsler Intelligence Scale for Children, Fourth Edition (WISC-IV) [35] according to the patient's age to assess their intelligence level. Further, the Mini International Neuropsychiatric Interview (M.I.N.I.) [31] will be used to screen the parents of participating patients for the presence of diagnostic criteria, including panic disorder, agoraphobia, general anxiety, and depression.

\section{Outcome measures Primary outcomes}

Autism Knowledge Quiz-Child (AKQ-C) AKQ-C [17] has two sections, namely, a five-item structured interview on "awareness of autistic characteristics" and a 15item knowledge quiz. The main evaluation items of this study include variations in the "awareness of autistic characteristics" measured in section 1 of the AKQ-C, which will be administered during the pre-intervention, post-intervention, and follow-up period.

Table 2 Agenda for each session

\begin{tabular}{ll}
\hline Minutes (total 100) & Contents of each session \\
\hline 10 & Check the condition and mood over the week (0-100 evaluation) based on the ACAT textbook \\
20 & Check homework assignments \\
20 & Read the ACAT textbook related to the day's topic (about the day's topic, see Table 1) \\
10 & Break \\
30 & Practice work (e.g., cognitive and behavioral intervention) related to the day's topic based on the ACAT textbook \\
5 & Set homework assignment that is related to the day's topic \\
5 & Ask the patient and parent for feedback about the session \\
\hline
\end{tabular}


Table 3 Standard Protocol Items: Recommendations for Interventional Trials (SPIRIT) diagram

\begin{tabular}{|c|c|c|c|c|c|c|c|}
\hline \multirow[t]{3}{*}{ Time point ${ }^{* *}$} & \multicolumn{7}{|l|}{ Study period } \\
\hline & \multirow{2}{*}{$\begin{array}{l}\text { Enrollment, } \\
\text { May } 2017_{1}\end{array}$} & \multirow{2}{*}{$\begin{array}{l}\text { Allocation, } \\
\text { March } \\
2018\end{array}$} & \multicolumn{4}{|c|}{ Post-allocation } & \multirow{2}{*}{$\begin{array}{l}\text { Close- } \\
\text { out, } \\
\text { May } \\
2020\end{array}$} \\
\hline & & & Ow & $6 w$ & $10 w$ & etc. & \\
\hline \multicolumn{8}{|l|}{ Enrollment: } \\
\hline Eligibility screen & $x$ & & & & & & \\
\hline Informed consent & $x$ & & & & & & \\
\hline Allocation & & $x$ & & & & & \\
\hline \multicolumn{8}{|l|}{ Interventions: } \\
\hline Combination (ACAT) group & & & $x$ & $x$ & $x$ & & \\
\hline$T A U$ group & & & $x$ & $x$ & $x$ & & \\
\hline \multicolumn{8}{|l|}{ Assessments: } \\
\hline Socioeconomic and demographic data & X & $x$ & $x$ & $x$ & $x$ & & \\
\hline Patients: AKQ-C, DSRS-C, BACEv3, EEG & $x$ & $x$ & $x$ & $x$ & $x$ & & \\
\hline Parents: AKQ-P, SDQ, Vineland-2, Bace-v3, GHQ-12, EEG & $x$ & $x$ & $x$ & $x$ & $x$ & & \\
\hline
\end{tabular}

AKQ-C Autism Knowledge Quiz-Child, DSRS-C Depression Self-Rating Scale for Children, BACEv3 Barriers to Access to Care Evaluation scale version 3, EEG electroencephalogram, AKQ-P Autism Knowledge Quiz-Parents, SDQ Strengths and Difficulties Questionnaire, GHQ-12 General Health Questionnaire

\section{Secondary outcomes}

Depression Self-Rating Scale for Children (DSRS-C) DSRS-C [36] is a self-assessment scale containing 18 items. It assesses the level of depression among children by enquiring about their feelings over a week; each item is marked on a three-point scale: "always," "sometimes," and "never." This scale will be applied to children/adolescents at three different time points: pre-intervention, post-intervention, and follow-up.

Barriers to Access to Care Evaluation scale version 3 (BACE-3) The BACE [37] is a 30-item questionnaire designed to assess the barriers to mental health care for people with mental health problems. It includes barriers related and unrelated to stigma and discrimination. This scale will be applied to both children/adolescents and parents/guardians at three different time points: preintervention, post-intervention, and follow-up.

Strengths and Difficulties Questionnaire (SDQ) SDQ [30] is a short screening instrument that addresses the positive and negative behavioral attributes of infants, children, and adolescents. It includes 25 items, and each item can be marked "not true," "somewhat true," or "certainly true." The scale will be applied to parents/guardians who are requested to score their children/ adolescents at three different time points: preintervention, post-intervention, and follow-up.

Vineland Adaptive Behavior Scales second edition (Vineland-2) Vineland-2 [38] is a semi-structured interview for parents to capture the developmental norm of adaptive behavior in individuals aged 0-92 years. Interviews will be conducted with parents/guardians about their children at three different time points: preintervention, post-intervention, and follow-up.

Autism Knowledge Quiz-Parents (AKQ-P) (for parents/guardians) AKQ-P [17] is the parent version of AKQ-C. We will apply this scale to parents/guardians at three different time points: pre-intervention, postintervention, and follow-up.

The 12-item General Health Questionnaire (GHQ12) (for parents/guardians) GHQ [39] is a measure of current mental health. The questionnaire was originally developed as a 60-item instrument; however, a range of shortened versions of the questionnaire such as GHQ30, GHQ-28, GHQ-20, and GHQ-12 are available. The scale tests if respondents recently experienced a particular symptom or behavior. Each item is rated on a 4point scale (less than usual, no more than usual, rather more than usual, or much more than usual); for GHQ12, it gives a total score of 36 or 12 based on the selected scoring methods. We will apply this scale to parents/ guardians at three different time points: preintervention, post-intervention, and follow-up.

Parenting Resilience Elements Questionnaire (PREQ) (for parents/guardians) PREQ [40] measures the degree to which mothers possess elements that aid in adapting to challenges and difficulties related to children with developmental disorders. It comprises 29 items and three factors, namely, "knowledge of the child's characteristics, " "perceived social supports," and "positive perceptions 
of parenting." Each item is scored on a 7-point Likert scale from 1 (strongly disagree) to 7 (strongly agree). We will apply this scale to parents/guardians at three different time points: pre-intervention, post-intervention, and follow-up.

Electroencephalogram (EEG) An EEG analysis can be used to detect the disrupted functions of large-scale cortical activity in ASD patients and to provide objective indices to evaluate the effectiveness of this program [41]. Therefore, resting-state EEG will be recorded as an objective physiological index alongside the questionnaire. The EEG scale will be applied to both children/adolescents and parents/guardians at three different time points: pre-intervention, post-intervention, and follow-up.

\section{Other measurements}

Before and after treatment, parents are requested to report their children's current medication, dose of medication, and outpatient (hospital) care.

\section{Sample size}

The sample size was calculated using the $\mathrm{G}^{*}$ power software version 3.1.9.2 with a power of 0.80 and $\alpha=0.05$ (two-tailed). In a previous study [17], the change in AKQ's "ASD awareness" was 2.26, and the effect size was 0.92 . Considering a drop out ratio of $20 \%$, the ideal sample size was estimated as 48 .

\section{Statistical methods}

\section{Statistical analysis plan}

We are currently collecting data from 41 of 48 child/adolescent ASD patients and their parents/guardians. Treatments will end during the spring of 2020, and analysis of the data will commence in the summer of 2020. The purpose of this study is to examine the relative efficacy of ACAT for child/adolescent ASD patients and their parents/guardians, in terms of ASD awareness scores, compared to the TAU group. Therefore, the primary analysis will evaluate the differences in score variations (i.e., the ACAT and TAU groups' mean variations) for ASD awareness from week 0 (baseline) to week 6 . Relative efficacy will be indicated when the point estimate of mean variation difference has a positive value and when the value of the ACAT group statistically exceeds that of the TAU group. The examination of scores will be based on an analysis of covariance (ANCOVA) for interval estimation of mean variation differences and calculation of the $P$ value using allocation factors as adjustment factors (gender and AKQ-C score). The secondary evaluation of efficacy items will supplement the main results of the analysis, and redundancy will not be adjusted. The level of significance for the hypothesis test is $5 \%$ on two sides, with a twosided confidence interval of $95 \%$.
The results of the BACE-3, AKQ-P, SDQ, Vineland-2, DSRS-C, PREQ, and GHQ-12 (self-completed by parents) will be analyzed using the same techniques used for the assessment of the main evaluation items. In addition, EEG of individuals with HF-ASD as well as their parents/guardians will be recorded at pre-intervention, postintervention, and follow-up to investigate neurological changes. EEG signals will be registered with a 19-channel box of Mitsar-EEG 201 (Mitsar Co. Ltd.). The 19-channel EEG cap will be fitted in accordance with a standard 1020 system. EEG analysis will be conducted based on graph theory. The overall efficiency, clustering coefficients, connection between the left and right hemispheres, and network resilience will be calculated and compared between the ACAT and TAU groups.

\section{Discussion}

Children diagnosed with autism are typically portrayed as being significantly different from other children. The experiences of children diagnosed with autism are defined as either positive or negative [42]. Conversely, it has been reported that being diagnosed with ASD in the absence of aftercare avenues, including psychoeducation and assistance in increasing self-understanding, diminishes the benefit of a diagnosis [13, 17]. A diagnosis should deepen one's understanding of ASD characteristics, and psychoeducation for ASD is crucial to continued understanding and awareness. This must follow a diagnosis to prevent the amplification of related psychological problems such as the perception of self-stigma and lowered self-esteem.

We expect that participants and parents assigned to the ACAT group who experience ACAT will gain a significantly greater understanding of ASD compared to those of the TAU group. Along with an enhanced comprehension of ASD, participants in the ACAT group are also expected to display improved social adaptation, decreased perception of self-stigma, and a reduction in depressive symptoms. Furthermore, the parents of participants are expected to experience less self-stigma, improved mental health, and reinforcement of nurturing resilience. A better understanding of a participant's ASD characteristics may lead to improved mental health in parents and encourage adaptive behaviors in the participants. Importantly, Gordon et al. [17] reported no significant changes in participants' self-esteem as a result of an enhanced understanding of ASD. This study assumes that improved self-esteem is experienced after increased social adaptation. However, there was no significant improvement in self-esteem in their study. ACAT may help to increase social adaptation through psychoeducation combined with CBT. We hope that the results of this study will support the benefits of a HF-ASD diagnosis in Japan. Some limitations should be noted, however. First, 
the comparative effectiveness of the psychoeducation phases of the PEGASUS and ACAT programs is unclear because of the differences in their components. This study is not designed to compare the psychoeducation phases of PEGASUS and ACAT. Hence, we cannot disentangle the specific effects of the psychoeducation phase used in the ACAT program. Second, ACAT is designed as family CBT, and our study design does not include individual CBT as a control group. Individual CBT dealing with problems related to autistic traits may be as effective as ACAT; nevertheless, it is expected that ACAT will be more acceptable and effective for younger participants. Further studies are warranted to establish the effectiveness of these treatment methods.

In conclusion, we expect that participants in the ACAT group will experience significantly increased selfawareness and understanding of ASD symptoms compared to those in the TAU group. We also expect the ACAT group to experience significantly improved social adaptation and mental health if the children and parents in this group gain an adaptive comprehension of their own autistic traits during the sessions. This intervention may contribute to the establishment of an effective treatment strategy based on the evidence gleaned from adolescents with ASD.

\section{Trial status}

The protocol version number

May 30, 2017, version 1.0 (first edition); August 20, 2017, 2.0 edition; September 25, 2017, 3.0 edition; February 19, 2018, 3.5 edition; March 18, 2018, 4.0 edition; April 18, 2019, 4.5 edition; September 5, 2019, 5 edition

The date recruitment began: January 06, 2018

The approximate date when recruitment will be completed: May 2020.

\footnotetext{
Abbreviations

ACAT: Aware and Care for my Autistic Traits; ADOS-2: Autism Diagnostic Observation Schedule Second Edition; ASD: Autism spectrum disorder; ADIR: Autism Diagnostic Interview-Revised; ADHD-RS: ADHD Rating Scale-IV; AKQ: Autism Knowledge Quiz; AKQ-C: Autism Knowledge Quiz-Child; BACE3: Barriers to Access to Care Evaluation scale version 3; CBT: Cognitivebehavioral therapy; COMB: Combination treatment group; CONSORT: Consolidated Standards of Reporting Trials; DSRS-C: Depression Self-Rating Scale for Children; EEG: Electroencephalogram; GHQ-12: The 12item General Health Questionnaire; HF-ASD: High-functioning ASD; IQ: Intelligence quotient; M.I.N.I.: Mini-International Neuropsychiatric Interview; PACT: Parent-mediated communication-focused treatment in children with autism; PEGASUS: PsychoEducational Groups for Autism Spectrum Understanding and Support; PREQ: Parenting Resilience Elements Questionnaire; SDQ: Strengths and Difficulties Questionnaire; SPIRIT: Standard and Protocol Items: Recommendations for Interventional Trials; TAU: Treatment-as-usual; Vineland-2: Vineland Adaptive Behavior Scales second edition; WAIS-III: Wechsler Adult Intelligence Scale-III; WISCIV: Wechsler Intelligence Scale for Children, Fourth Edition
}

\section{Acknowledgements}

Our deep appreciation is extended to Kanae Osumi at the Hamamatsu University School of Medicine for her immense contribution to this investigation.

\section{Study status}

This is an ongoing clinical trial.

\section{Related articles}

There are no publications containing the results of this study.

\section{Authors' contributions}

Study concept and design: FO, KS, NT, and AT. Statistical analysis for study design: YK, YO, and YT. Drafting of the manuscript: FO, AT, YH, TO, DM, and HK. Critical revision of the manuscript: AS, HK, ES, and AN. Study therapists: $\mathrm{MH}, \mathrm{Yl}, \mathrm{TO}, \mathrm{NS}, \mathrm{SN}, \mathrm{MK}$, and JT. Study supervision: NI, MK, MA, AN, ES, and HK. All the authors have approved the submitted final version and any substantially modified version that involves the author's contribution to the study. All authors have agreed to be personally accountable for the author's own contributions and to ensure that questions related to the accuracy or integrity of any part of the work, even the ones in which the author was not personally involved are appropriately investigated, resolved, and the resolution documented in the literature.

\section{Funding}

The study was supported by the special research grant (No. 2017 S-1) of the Association of Japanese Clinical Psychology, which is a non-profit association for clinical psychology in Japan. The funders had no role in the design of the study; collection, analysis, and interpretation of the data; or in writing the manuscript.

This is an on-going research, and we have therefore not completed the data collection; hence, there is no outcome yet.

\section{Availability of data and materials}

The datasets generated and analyzed during the current study are not publicly available because analysis during research is prohibited; however, they can be made available upon reasonable request through the corresponding author.

\section{Ethics approval and consent to participate}

This trial was approved by the Chiba University Clinical Research Ethical Review Committee (CRB3180015, No. G29027). Written informed consent was obtained from the participating patients and their parents by providing a consent form approved by the Chiba University Clinical Research Ethical Committee (CREC), following a verbal explanation of the trial along with the consent form. Any personal information (including anonymized data) is treated as confidential. Participants and their parents or guardians are able to access these data after follow-up upon request. This clinical trial was registered on the University Hospital Medical Information Network (UMIN) Center (No. 000029851). The CREC reviewed this protocol. Amendments or changes to the protocol will be referred to the CREC and will be updated on the UMIN.

\section{Consent for publication}

Not applicable

\section{Competing interests}

The authors have no conflicts of interest to declare relevant to the content of this paper.
Author details
${ }^{1}$ Research Center for Child Mental Development, Chiba University, 1-8-1 Inohana Chuouku, Chiba 260-8670, Japan. ${ }^{2}$ Research Department of Clinical, Educational \& Health Psychology, University College London, London, UK. ${ }^{3}$ Fukushima University Child Mental Health-Care Center, Fukushima, Japan. ${ }^{4}$ Laureate Instituto for Brain Research, Tulsa, OK, USA. ${ }^{5}$ Department of Psychiatry, Hamamatsu University School of Medicine, Shizuoka, Japan. ${ }^{6}$ Chiba University Center for Forensic Mental Health, Chiba, Japan. ${ }^{7}$ Biostatistics Section, Clinical Research Center, Chiba University Hospital, Chiba, Japan. ${ }^{8}$ Department of Psychiatry, Tokyo Medical University lbaraki Medical Center, Ibaraki, Japan. ${ }^{9}$ Department of Psychology, Faculty of Liberal Arts, Teikyo University, Tokyo, Japan. ${ }^{10}$ Department of Human Care, Nagoya University of Arts and Sciences, Nagoya, Japan. " ${ }^{11}$ epartment of Psychiatry, Hibarigaoka Hospital, Fukushima, Japan. ${ }^{12}$ Hori Mental Clinic, Fukushima, Japan. 
Received: 19 March 2020 Accepted: 17 September 2020

Published online: 29 September 2020

\section{References}

1. American Psychiatric Association. Diagnostic and statistical manual of mental disorders. 5th ed. Washington DC: Publisher; 2013

2. Kamio $Y$, Inada N, Koyama T. A nationwide survey on quality of life and associated factors of adults with high-functioning autism spectrum disorders. Autism. 2013;17:15-26.

3. Zablotsky B, Black LI, Maenner MJ, Schieve LA, Blumberg SJ. Estimated prevalence of autism and other developmental disabilities following questionnaire changes in the 2014 National Health Interview Survey. Natl Health Stat Rep. 2015:87:1-20.

4. Gray DE. 'Everybody just freezes. Everybody is just embarrassed': felt and enacted stigma among parents of children with high functioning autism. Sociol Health IIIn. 2002;24:734-49.

5. White SW, Oswald D, Ollendick T, Scahill L. Anxiety in children and adolescents with autism spectrum disorders. Clin Psychol Rev. 2009;29:216-29.

6. Weiss JA, Lunsky Y. Group cognitive behaviour therapy for adults with Asperger syndrome and anxiety or mood disorder: a case series. Clin Psychol Psychother. 2010;17:438-46.

7. Ekman E, Hiltunen AJ. Modified CBT using visualization for autism spectrum disorder (ASD), anxiety and avoidance behavior--a quasi-experimental open pilot study. Scand J Psychol. 2015;56(6):641-8. https://doi.org/10.1111/sjop.12255.

8. Rogers SJ, Estes A, Lord C, Vismara L, Winter J, Fitzpatrick A, et al. Effects of a brief Early Start Denver Model (ESDM)-based parent intervention on toddlers at risk for autism spectrum disorders: a randomized controlled trial. J Am Acad Child Adolesc Psychiatry. 2012;51:1052-65.

9. Green J, Charman T, McConachie H, Aldred C, Slonims V, Howlin P, et al. Parent-mediated communication-focused treatment in children with autism (PACT): a randomised controlled trial. Lancet. 2010;375:2152-60.

10. Choque Olsson N, Flygare O, Coco C, Görling A, Råde A, Chen Q, et al. Social skills training for children and adolescents with autism spectrum disorder: a randomized controlled trial. J Am Acad Child Adolesc Psychiatry. 2017:56:585-92

11. Gantman A, Kapp SK, Orenski K, Laugeson EA. Social skills training for young adults with high-functioning autism spectrum disorders: a randomized controlled pilot study. J Autism Dev Disord. 2012;42(6):1094-103. https://doi. org/10.1007/s10803-011-1350-6.

12. Sarrett JC. Autism and accommodations in higher education: insights from the autism community. J Autism Dev Disord. 2018:48:679-93.

13. Calzada LR, Pistrang N, Mandy WPL. High-functioning autism and Asperger's disorder: utility and meaning for families. J Autism Dev Disord. 2012:42:230-43.

14. Lucksted A, McFarlane W, Downing D, Dixon L. Recent developments in family psychoeducation as an evidence-based practice. J Marital Fam Ther. 2012;38:101-21.

15. Midence $\mathrm{K}, \mathrm{O}^{\prime} \mathrm{Neill} M$ M. The experience of parents in the diagnosis of autism: a pilot study. Autism. 1999;3:273-85

16. Farrugia D. Exploring stigma: medical knowledge and the stigmatisation of parents of children diagnosed with autism spectrum disorder. Sociol Health IIIn. 2009;31:1011-27.

17. Gordon K, Murin M, Baykaner O, Roughan L, Livermore-Hardy V, et al. A randomised controlled trial of PEGASUS, a psychoeducational programme for young people with high-functioning autism spectrum disorder. J Child Psychol Psychiatry. 2015;56:468-76.

18. Beck AT. Cognitive therapy: nature and relation to behavior therapy. Behav Ther. 1970;1:184-200.

19. Russell AJ, Jassi A, Fullana MA, Mack H, Johnston K, Heyman I, et al. Cognitive behavior therapy for comorbid obsessive-compulsive disorder in high-functioning autism spectrum disorders: a randomized controlled trial. Depress Anxiety. 2013;30:697-708.

20. Gaus VL. Cognitive-behavioral therapy for adult Asperger syndrome. New York: Guilford Press; 2007.

21. Sofronoff K, Eloff J, Sheffield J, Attwood T. Increasing the understanding and demonstration of appropriate affection in children with Asperger syndrome: a pilot trial. Autism Res Treat. 2011;2011:214317.

22. Wood JJ, Drahota A, Sze K, Marilyn VD, DykeKelly D, Cori F, et al. Brief report: effects of cognitive behavioral therapy on parent-reported autism symptoms in school-age children with high-functioning autism. J Autism Dev Disord. 2009;39:1608.
23. Neuhaus E, Beauchaine TP, Bernier R. Neurobiological correlates of social functioning in autism. Clin Psychol Rev. 2010;30:733-48.

24. Barttfeld P, Wicker B, Cukier S, Navarta S, Lew S, Sigman M. A big-world network in ASD: dynamical connectivity analysis reflects a deficit in longrange connections and an excess of short-range connections. Neuropsychologia. 2011;49(2):254-63.

25. Chan AW, Tetzlaff JM, Altman DG, Laupacis A, Gøtzsche PC, Krleža-Jerić K, et al. SPIRIT 2013 Statement: defining standard protocol items for clinical trials. Ann Intern Med. 2013;158:200-7.

26. Moher D, Hopewell S, Schulz KF, Montori V, Gøtzsche PC, Devereaux PJ, et al. CONSORT 2010 explanation and elaboration: updated guidelines for reporting parallel group randomised trials. Int J Surg. 2012;10:28-55.

27. Lord C, Rutter M, DiLavore PC, Risi S. Autism diagnostic observation schedule-WPS (ADOS-WPS). Western Psychological Services: Los Angeles; 1999.

28. Lord C, Rutter M, Le Couteur A. Autism diagnostic interview-revised: a revised version of a diagnostic interview for caregivers of individuals with possible pervasive developmental disorders. J Autism Dev Disord. 1994;24: 659-85.

29. Seashore H, Wesman A, Doppelt J. The standardization of the Wechsler Intelligence Scale for Children. J Consult Psychol. 1950;14:99-110.

30. Goodman R. The strengths and difficulties questionnaire: a research note. J Child Psychol Psychiatry. 1997;38:581-6.

31. Sheehan D, Lecrubier Y, Harnett Sheehan K, Janavs J, Weiller E, Keskiner A, et al. The validity of the Mini International Neuropsychiatric Interview (MINI) according to the SCID-P and its reliability. Eur Psychiatry. 1997;12:232-41.

32. Young JE, Beck AT. 1980. https://beckinstitute.org/.

33. DuPaul GJ, Power TJ, Anastopoulos AD, Reid R. ADHD rating scale-IV: checklists, norms, and clinical interpretation. New York: Guilford; 1998.

34. Winnie D. Sensory profile. London: Pearson; 1999.

35. Kaufman AS, Flanagan DP, Alfonso VC, Mascolo JT. Test review: Wechsler Intelligence Scale for Children, fourth edition (WISC-IV). J Psychoeduc Assess. 2006;24:278-95.

36. Birleson P. The validity of depressive disorder in childhood and the development of a self-rating scale: a research report. J Child Psychol Psychiatry. 1981;22:73-88.

37. Clement S, Brohan E, Jeffery D, Henderson C, Hatch SL, Thornicroft G. Development and psychometric properties the Barriers to Access to Care Evaluation scale (BACE) related to people with mental ill health. BMC Psychiatry. 2012;12:36.

38. Sparrow SS, Cicchetti DV, Balla D. Vineland Adaptive Behavior Scales, second edition: survey interview forms manual. 2nd ed. Minneapolis: NCS Pearson Inc; 2005.

39. Montazeri A, Harirchi AM, Shariati M, Garmaroudi G, Ebadi M, Fateh A. The 12-item General Health Questionnaire (GHQ-12): translation and validation study of the Iranian version. Health Qual Life Outcomes. 2003;1:66.

40. Suzuki K, Kobayashi T, Moriyama K, Kaga M, Hiratani M, Watanabe K, et al. Development and evaluation of a Parenting Resilience Elements Questionnaire (PREQ) measuring resiliency in rearing children with developmental disorders. PLoS One. 2015;10:e0143946.

41. Jia H, Yu D. Aberrant intrinsic brain activity in patients with autism spectrum disorder: insights from EEG microstates. Brain Topogr. 2019;32:295-303.

42. Wright A, Jorm AF, Mackinnon AJ. Labeling of mental disorders and stigma in young people. Soc Sci Med. 2011;73:498-506.

\section{Publisher's Note}

Springer Nature remains neutral with regard to jurisdictional claims in published maps and institutional affiliations.

\section{Ready to submit your research? Choose BMC and benefit from:}

- fast, convenient online submission

- thorough peer review by experienced researchers in your field

- rapid publication on acceptance

- support for research data, including large and complex data types

- gold Open Access which fosters wider collaboration and increased citations

- maximum visibility for your research: over $100 \mathrm{M}$ website views per year

At $\mathrm{BMC}$, research is always in progress.

Learn more biomedcentral.com/submission 